\title{
Spontaneous Esophageal Perforation and Hypopharyngeal Abscess in Adult Dermatomyositis: A Case Report
}

\author{
Min Kim', Song Yi Choi ${ }^{2}$, Seung Young Lee ${ }^{3}$, Kwi Young Kang ${ }^{4}$ \\ Departments of Internal Medicine ${ }^{1}$, Pathology ${ }^{2}$, Radiology $^{3}$, \\ Chungbuk National University Hospital, Cheongju, Department of Internal Medicine, \\ Incheon Saint Mary's Hospital, The Catholic University of Korea, Incheon, Korea
}

In patients with dermatomyositis, chronic inflammation of the pharynx and esophagus results in coughing and difficulty in swallowing. These become important clinical symptoms, especially if they contribute to malnutrition or aspiration pneumonia. They can ultimately reduce the quality of life. In rare cases, if the symptoms worsen despite proper treatment, serious complications may arise, a reason to suspect an esophageal perforation or abscess.

\section{Introduction}

Dermatomyositis (DM) is thought to represent a complement-mediated small vessel vasculopathy, and a chronic inflammatory myopathy that frequently affects the skin, muscles and lungs $(1,2)$. Pharyngeal muscle weakness may contribute to dysphagia, dysphonia and aspiration pneumonia, causing life-threatening complications, and decreased quality of life. Hypopharyngeal abscess and spontaneous esophageal perforation are very rare conditions of dermatomyositis and have a poor prognosis, with a 1 year mortality rate of approximately $31 \%(3,4)$. A delay in diagnosis and treatment leads to worse outcomes of these potentially life-threatening complications. In this case report, we present a DM patient who developed a spontaneous esophageal perforation and hypopharyngeal abscess.

\section{Case Report}

A 55-year-old man with a 2 month history of proximal muscle weakness and dry cough was admitted because of chest
The authors report a case of dermatomyositis in an adult patient with rare complications of spontaneous esophageal perforation and hypopharyngeal abscess. The patient received non-surgical treatment and was able to resume oral intake of food.

Key Words. Dermatomysitis, Hypopharynx, Esophagus, Perforation, Abscess

pain on July, 2011. He also had a heliotrope rash, and reported recent solid food dysphagia and dysphonia. Laboratory tests revealed serum creatine phosphokinase (CPK) of 4,777 IU/L, myoglobin $1,167 \mathrm{ng} / \mathrm{mL}$, lactate dehydrogenase 2,214 $\mathrm{IU} / \mathrm{L}$, erythrocyte sedimentation rate (ESR) $55 \mathrm{~mm} / \mathrm{h}, \mathrm{C}$ reactive protein (CRP) $0.58 \mathrm{mg} / \mathrm{dL}$, hemoglobin $14.6 \mathrm{~g} / \mathrm{dL}$, hematocrit $43.3 \%$, white blood cell count $5,500 / \mathrm{LL}$, platelet 180/uL, AST 267 IU/L, ALT 242 IU/L, alkaline phosphatase (ALP) $161 \mathrm{IU} / \mathrm{L}$, total bilirubin $0.17 \mathrm{mg} / \mathrm{dL}$, blood urea nitrogen at $9.3 \mathrm{mg} / \mathrm{dL}$ and serum creatinine at $0.6 \mathrm{mg} / \mathrm{dL}$. Antinuclear antibodies were positive and anti-Jo-1 antibodies were negative. The muscle power grade of the upper limbs and lower limbs were grade III. Electromyography findings included positive sharp waves, fibrillations, and small amplitude that were consistent with myopathy. A muscle biopsy showed focal perifascicular atrophy, perivascular infiltration of predominantly $\mathrm{CD} 4+$ lymphohistiocytes, and degenerating and regenerating changes consistent with a diagnosis of DM (Figure 1). Computed tomography (CT) of the chest showed

\footnotetext{
<Received: October 4, 2012, Revised (1st: January 22, 2013, 2nd: February 18, 2013), Accepted : February 18, 2013> Corresponding to : Kwi Young Kang. Division of Rheumatology, Department of Internal Medicine, Incheon St. Mary's Hospital, The Catholic University of Korea, 56, Dongsu-ro, Bupyeong-gu, Incheon 403-720, Korea. E-mail : kykang@ catholic.ac.kr
} 

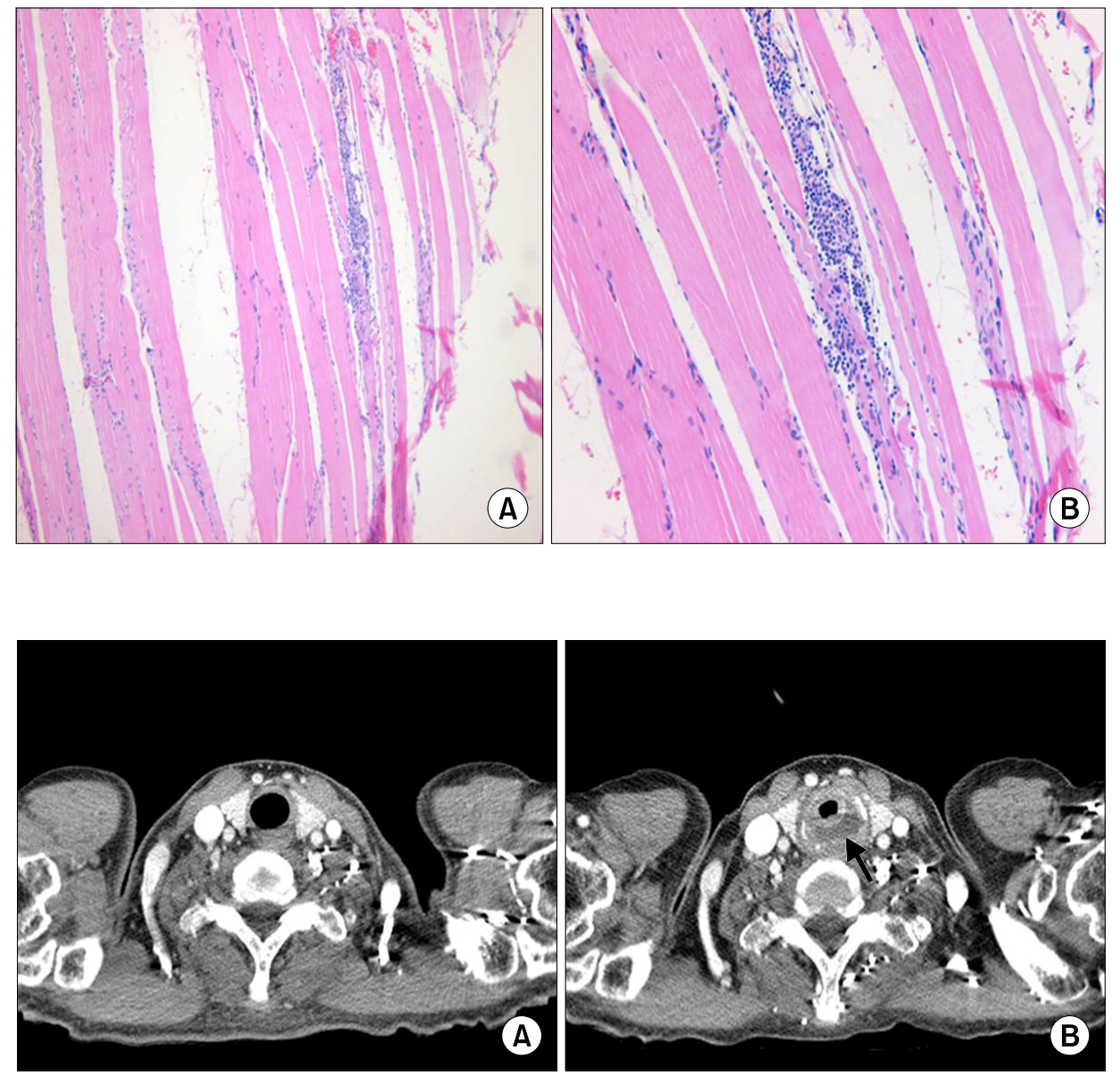

Figure 1. (A) Histopathologic features of an incisional biopsy of the vastus lateralis muscle revealed variation in the fiber sizes and focal perifascicular atrophy, $\times 40$. (B) Muscle fibers showing degenerating and regenerating changes and perivascular infiltration of lymphohistiocytes, $\times 200$.

Figure 2. (A) Computed tomography of the chest showed no remarkable findings in the pharynx in July, 2011. (B) Computed tomography of the chest showed peripheral enhancing fluid collection in the inferior aspect of cricoids cartilage, suggesting the presence of an abscess formation in December, 2011 (black arrow) interstitial lung disease and no pharyngeal abnormalities (Figure 2A). On screening evaluation for malignancy, CT of the abdomen and pelvis, as well as esophagoduodenoscopy and colonofiberoscopy, were all normal.

Videofluoroscopic study (VFS) revealed pharyngeal pooling and aspiration. During hospitalization, he was kept on alimentary abstinence. A nasogastric tube was inserted carefully for feeding, and intravenous methylprednisolone $(62.5 \mathrm{mg} / \mathrm{d})$ was started. In addition, he received Azathioprine ( $50 \mathrm{mg} /$ day). On admission day 23 , he started complaining of a productive cough and fever. Chest X-ray showed haziness in the right lower lung field, and clinical examination revealed aspiration pneumonia. Azathioprine was stopped, and he was started on empirical antibiotic treatment. Pharyngeal muscle function did not recover with methylprednisolone, so he was started on intravenous gamma-immunoglobulin (20 g/day, 5 days). Proximal muscle weakness began to improve, and the grades of muscle power increased to grade $\mathrm{V}$ in the upper limbs and grade IV in the lower limbs. Methylprednisolone was gradually tapered to prednisolone $(25 \mathrm{mg} /$ day $)$ with addition of methotrexate $(10 \mathrm{mg} /$ week). The muscle weakness gradually resolved but despite slight improvement on VFS, he was not able to resume a normal oral diet. He was discharged with a nasogastric tube. One month later, he was able to resume a soft blend oral diet carefully following an additional VFS.

In December, 2011, he was re-admitted because of a productive cough and worsened dysphagia, without fever. Laboratory tests revealed a serum CPK of 145 IU/L, ESR 57 $\mathrm{mm} / \mathrm{hr}$, and CRP $0.45 \mathrm{mg} / \mathrm{dL}$. Unlike the previous imaging, CT of the chest showed mucosal enhancement involving the hypopharynx above the pyriform sinus, and fluid collection at the inferior aspect of the cricoid cartilage, suggesting the presence a of deep neck infection with abscess formation (Figure 2B). Barium esophagography revealed contrast media leakage, which suggested esophageal perforation (Figure 3A). Surgery was considered, but it was decided to attempt medical treatment first because his vital signs were stable and his general condition was not worsening. He was kept on alimentary abstinence and was started on empirical antibiotics. After 3 weeks of follow-up, esophageal leakage was no longer found on esophagography (Figure 3B), and CT of the neck showed a decreased amount of fluid collection. VFS showed normal 


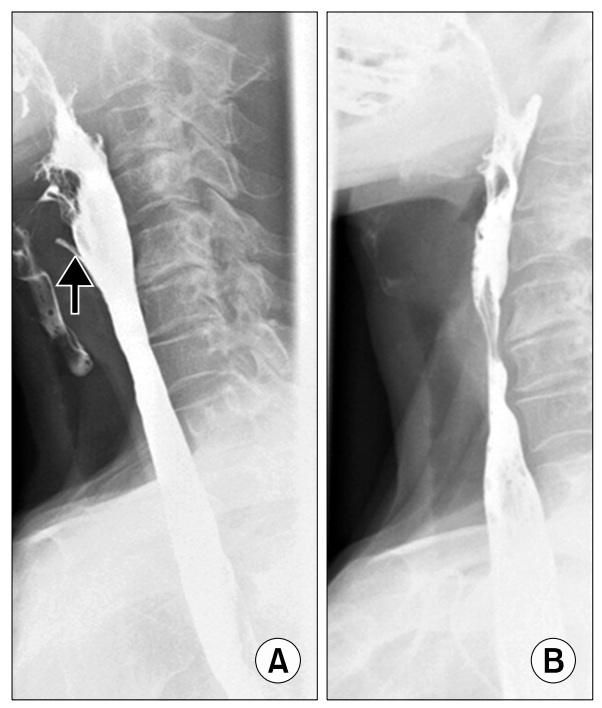

Figure 3. (A) Esophagography, revealing the contrast media leakage from the esophagus at the anteriosuperior level of the cricoid cartilage, suggesting an esophageal perforation in December, 2011 (black arrow). (B) Esophagography showing no evidence of the contrast media leakage after 1 month.

swallowing, and improvement of dysphagia. He was able to resume a soft blend diet carefully. He was discharged with oral antibiotics and prednisolone $7.5 \mathrm{mg}$ /day.

\section{Discussion}

DM is an uncommon chronic inflammatory disease, characterized by a proximal muscle weakness and skin rash. Approximtely $32 \sim 84 \%$ of patients with DM have associated involvement of the esophagus and pharynx, with frequent involvement of the esophageal sphincter muscle (2). The involvement of the pharynx and esophagus may result in a perforation or a fistula. Dysphagia is reported to have a 1 year mortality rate of $31 \%$ (4). There have been previous reported cases of spontaneous tracheoesophageal fistula resulting from vasculitis in adults with DM (5). Pneumoencephalon has also been reported in an adult DM because of a subarachnoid cervical fistula (1). Chronic inflammation-induced continuous mucosal injury and laceration caused the fistula. As with this patient, spontaneous esophageal rupture has also been reported in adult DM (6) as well as in chronic infections, such as tuberculosis (7) or candida infection (8). In those cases, the etiology of the disease was chronic inflammation and necrosis. In our case, the adult patient with DM experienced spontaneous esophageal perforation and hypopharyngeal abscess at the same time. Aspiration pneumonia occurred while the patient was on alimentary abstinence and had a nasogastric tube. Given those circumstances, spontaneous esophageal perforation was likely to have existed on the first admission, but was not detected because of the continuous nasogastric feeding and alimentary abstinence. When the patient started to take in food orally, it is likely that the food and liquid contents caused chronic infection through the perforation site. The hypopharyngeal abscess could have developed as a result.

Regardless of the cause, esophageal perforation is a major life-threatening event. Surgical, nonoperative, and minimally invasive treatment options are available. Surgical treatments include primary closure, esophagectomy, exclusion and diversion, and drainage. Nonoperative treatments include prophylactic broad-spectrum antibiotics, airway maintenance, and oral hygiene. Minimally invasive techniques include endoscopic stenting, clipping, and video-assisted thoracoscopic surgery (9). There have been a few reported cases of surgical intervention for spontaneous esophageal rupture with DM (6), nonoperative treatments for tracheoesophageal fistula with DM (5), and subarachnoid cervical fistula in DM (1). We consulted with a thoracic surgeon regarding the need for surgical treatment. However, because of the high risk of surgery, the patient's immunocompromised status and stable general condition, nonoperative treatment was recommended and followed. The patient showed gradual improvement and esophageal leakage has stopped. As such, the treatment was considered successful.

\section{Summary}

The clinical manifestation of pharyngeal muscle involvement in DM is common, but abscess formation or esophageal perforations are uncommon. With occurrence of aspiration pneumonia while maintaining nasogastric feeding, or if continuous foreign body sensations continue after recovery of pharyngeal muscle function, physicians should consider complications, such as a perforation or abscess formation. If the patient's condition is stable, spontaneous esophageal perforation and hypopharyngeal abscess may improve, with proper pharmacological and conservative management.

\section{References}

1. Iking-Konert C, Ostendorf B, Jung G, Becker A, Schneider M. "Bubbles in the brain": an unusual complication of dermatomyositis. Ann Rheum Dis 2006;65: 550-1.

2. Ebert EC. Review article: the gastrointestinal complications of myositis. Aliment Pharmacol Ther 2010;31: 359-65.

3. Williams RB, Grehan MJ, Hersch M, Andre J, Cook IJ. Biomechanics, diagnosis, and treatment outcome in inflammatory myopathy presenting as oropharyngeal dysphagia. Gut 2003;52:471-8.

4. Oh TH, Brumfield KA, Hoskin TL, Stolp KA, Murray 
JA, Bassford JR. Dysphagia in inflammatory myopathy: clinical characteristics, treatment strategies, and outcome in 62 patients. Mayo Clin Proc 2007;82:441-7.

5. Du Y, Dai N, Yu H, Lu Z. Tracheoesophageal fistula: a rare complication of adult dermatomyositis. Eur $\mathbf{J}$ Dermatol 2008;18:347-8.

6. Dougenis D, Papathanasopoulos PG, Paschalis C, Papapetropoulos T. Spontaneous esophageal rupture in adult dermatomyositis. Eur J Cardiothorac Surg 1996;10:1021-3.
7. Ghandour Z, al Karawi MA, Mohamed AE. Spontaneous esophageal perforation: unusual presentation of tuberculosis. Endoscopy 1997;29:143-4.

8. Al-Shawwa B, D'Andrea L, Quintero D. Candida esophageal perforation and esophagopleural fistula: a case report. J Med Case Rep 2008;2:209.

9. Wu JT, Mattox KL, Wall MJ Jr. Esophageal perforations: new perspectives and treatment paradigms. J Trauma 2007;63:1173-84. 